\title{
On the Null Scrolls in Minkowski 3-space $E_{1}^{3}$
}

\author{
Rashad A. Abdel-Baky ${ }^{a, b}$ and Maryam T. Aldossary ${ }^{c}$ \\ ${ }^{a}$ Present address: Department of Mathematics, Sciences Faculty for Girls, King Abdulaziz University, P.O. Box \\ 126300, Jeddah 21352 SAUDI ARABIA. \\ ${ }^{b}$ Department of Mathematics, Faculty of Science, University of Assiut, Assiut 71516, EGYPT. \\ ${ }^{c}$ Department of Mathematics Girls College of Science University of Dammam, P.O. Box-12577 Dahran-31311,
} SAUDI ARABIA

\begin{abstract}
We study ruled surfaces with lightlike ruling in Minkowski 3-space which are said to be null-scrolls. Even that the result is a consequence of some well-known results involving the Gauss map, we give another approach to classify all null-scrolls under the condition $\Delta \boldsymbol{Y}=A \boldsymbol{Y}$ where $\Delta$ is the Laplace operator with respect to the first fundamental form and $A \in \mathbb{R}^{3 \times 3}$ the set of $3 \times 3$ real matrices.
\end{abstract}

Key words: Null frame, Lorentzian ruled surface, Gauss map.

\section{Introduction}

Let $\mathbf{Y}: M \rightarrow E^{3}$ be an isometric immersion of a surface in Euclidean 3-space. Denotes by $\mathbf{G}$ and $\Delta$, respectively, the Gauss map and the Laplacian operator of the surface $M \square$ with respect to the induced metric from that of $E^{3}$. Takahashi [1] proved that minimal surfaces and spheres are the only surfaces in $E^{3}$ satisfying the condition

$\Delta \mathbf{Y}=A \mathbf{Y}$,

where $A \in \mathbb{R}^{3 \times 3}$ the set of $3 \times 3$ real matrices. Garay [2] extended it to the hypersurfaces, that is, he studied the hypersurfaces in $E^{n+1}$. On the other hand, Baikoussis and Blair [3] studied ruled surfaces such that their Gauss maps satisfy

$\Delta \mathbf{G}=A \mathbf{G}$.

They showed that the only ones are planes and circular cylinders. Also, for the Lorentz version S. M. Choi ([4]) showed that the only ruled surfaces with non-null base curve in a 3-dimensional Minkowski space $E_{1}^{3}$ satisfying the condition (1.2) are locally the Euclidean plane, the Minkowski plane, the Lorentz hyperbolic cylinder, the Lorentz circular cylinderand the hyperbolic cylinder. Furthermore, L. J. Alias, A. Ferrandez, P.

Lucas and M. A. Merono [5] proved that the only ruled surfaces in $E_{1}^{3}$ with null rulings satisfying thecondition (1.2) are B-scrolls over null curves.

In this paper, we introduced Lorentzian surfaces with lightlike rulings in a Minkowski3-space $E_{1}^{3}$ which are said to be null scrolls. Then, by using the Laplacian operator with respect to non-degenerate first fundamental form, we showed that all Lorentzian surfaces with the mean curvatures $H$ and the Gaussian curvatures $K$ satisfying $H^{2}=K$ can be classified into three kinds, two of which have no counterparts in the 3dimensional Euclidean space. The important point to note here is the technique we have used.

\section{Preliminaries}

The Minkowski 3 -space $E_{1}^{3}$ is the real vector space $\mathbb{R}^{3}$ endowed with the standard flat Lorentzian metric given by

$$
<, .>=-d x_{1}^{2}+d x_{2}^{2}+d x_{3}^{2}
$$

where $\left(x_{1}, x_{2}, x_{3}\right)$ is a rectangular coordinate system in $E_{1}^{3}$. Since $\langle\ldots, .>$ is an indefinite metric, recall that a vector $\mathbf{x}$ in $E_{1}^{3}$ is spacelike, lightlike (null) or timelike if $\langle\mathbf{x}, \mathbf{x}>>0,<\mathbf{x}, \mathbf{x}>=0$ or $<$ $\mathbf{x}, \mathbf{x}><0$, respectively. Then, for $\mathbf{x} \in E_{1}^{3}$ the $\mathrm{p}$ norm is defined by $\|\mathbf{x}\|=\sqrt{|<\mathbf{x}, \mathbf{x}>|}$, then the vector $\mathbf{x}$ is called a spacelike unit vector if $\langle\mathbf{x}, \mathbf{x}\rangle=1$ and a timelikeunit vector if $\langle\mathbf{x}, \mathbf{x}\rangle=-1$. Similarly, an arbitrary curve $\boldsymbol{\alpha}=\boldsymbol{\alpha}(s)$ in $E_{1}^{3}$ can locally be spacelike, timelike or null (lightlike), if all of its velocity vectors $\boldsymbol{\alpha}^{\prime}$ are 
spacelike, timelikeor null (lightlike), respectively. Here and what follows the derivative with respect to $s$ is denoted by a dash over functional symbol.

Definition 2.1.([6]). A surface in a Minkowski 3-space is called a Lorentzian (timelike) surface if the induced metric on the surface is a Lorentz metric, i.e., the normal on the surface is a spacelike vector.

Lemma 2.1. In the Minkowski 3 -space $E_{1}^{3}$, the following properties are satisfied [6, 7]:

(i) Two timelike vectors are never orthogonal.

(ii) Two null vectors are orthogonal if and only if they are linearly dependent.

(iii) A timelike vector is never orthogonal to a null (lightlike) vector.

A basis $F=\{\mathbf{T}, \mathbf{X}, \mathbf{Z}\}$ of $E_{1}^{3}$ is called a (proper) null frame if it satisfies the following conditions [6-8]:

$\langle\mathrm{T}, \mathrm{T}\rangle=\langle\mathrm{X}, \mathrm{X}\rangle=0$,

$\langle\mathrm{T}, \mathrm{Z}\rangle=\langle\mathrm{X}, \mathrm{Z}\rangle=0$,

$$
\langle\mathbf{Z}, \mathbf{Z}\rangle=1=-\langle\mathbf{T}, \mathbf{X}\rangle \text {. }
$$

It is easy show that:

$\mathbf{T} \times \mathbf{X}=\mathbf{Z}, \quad \mathbf{T} \times \mathbf{Z}=\mathbf{T}, \quad \mathbf{Z} \times \mathbf{X}=\mathbf{X}$.

Let $\alpha=\alpha(s)$ be a null curve in $E_{1}^{3}$; that is, a smooth curve whose tangent vectors $\boldsymbol{\alpha}^{\prime}=\boldsymbol{\alpha}(s) ; \mathbf{T}=\boldsymbol{\alpha}^{\prime}$ for every $s \in \mathrm{I}$ are null. Then $\mathbf{T}$ is a null vector field along $\boldsymbol{\alpha}(s)$. Moreover, there exists a null vector field $\mathbf{X}=\mathbf{X}(s)$ along $\boldsymbol{\alpha}$ satisfying $1=-\langle\mathbf{T}, \mathbf{X}\rangle$. If we put $\mathbf{T} \times \mathbf{X}=\mathbf{Z}$; then we can obtain a (proper) null frame field $F=\{\mathbf{T}, \mathbf{X}, \mathrm{Z}\}$ along $\alpha=\alpha(s)$. In this case the pair $(\boldsymbol{\alpha}, F)$ is said to be a (proper) framed null curve. A framed null curve $(\alpha, F)$ satisfies the following, so called the Frenet equations [8]:

$\left(\begin{array}{l}\mathbf{T}^{\prime} \\ \mathbf{X}^{\prime} \\ \mathbf{Z}^{\prime}\end{array}\right)=\left(\begin{array}{ccc}k_{1} & 0 & k_{2} \\ 0 & -k_{1} & k_{3} \\ k_{3} & k_{2} & 0\end{array}\right)\left(\begin{array}{l}\mathbf{T} \\ \mathbf{X} \\ \mathbf{Z}\end{array}\right)$.

The function $k_{i}=k_{i}(s),(i=1,2,3)$ is called an $\mathrm{i}$-th curvature of the framed null curve. It follows from the fundamental theorem of ordinary differential equations that a framed null curve $(\alpha, F)$ is uniquely determined by the functions $k_{i}$ and the initial condition.

\subsection{Null Scrolls in $E_{1}^{3}$}

Let $(\boldsymbol{\alpha}, F)=(\boldsymbol{\alpha}(s), F(s))$ be a null curve with frame field $F=\{\mathbf{T}, \mathbf{X}, \mathbf{Z}\}$. A ruled surface is a surface swept out by a straight line $\mathbf{X}$ moving along a curve $\boldsymbol{\alpha}$. The various positions of the generating line $\mathbf{X}$ are called the rulings of the surface. Such a surface, thus has a parametrization in ruled form as follows:

$M: \mathbf{Y}(s, u)=\boldsymbol{\alpha}(s)+u \mathbf{X}(s), u \in \mathbb{R}$

is called a null scroll and denoted by $M$. The curve $\boldsymbol{\alpha}(s)$ is called the directrix of $\mathrm{M}$ and $\mathbf{X}(s)$ is a vector attached to the straight line generating the ruled surface. If the tangent plane is constant along each ruling, then the ruled surface is called a developable surface.

The remaining ruled surfaces are called skew surfaces. One can see that $M$ is a Lorentzian surface. Especially when $k_{1}(s)=0, k_{2}=k(s)$ and $k_{3}$ is constant, $M$ is called a B-scroll [7].

For the components $g_{i j}$ of the induced Lorentzian metric $<, .,>$ on $M$ from that of $E_{1}^{3}$ we denote by $\left(g^{i j}\right)$ (resp. $D$ ) the inverse matrix (resp. the determinant) of the matrix $g_{i j}$. Then, the Laplacian $\Delta$ on $M$ is given by [6]:

$$
\Delta=-\frac{1}{\sqrt{|D|}} \sum_{i, j}\left(\frac{\partial}{\partial x_{i}} \sqrt{|D|} g^{i j} \frac{\partial}{\partial x_{j}}\right) .
$$

The Gaussian and mean curvatures of the Lorentzian surface $M$ in $E_{1}^{3}$, respectively, are

$$
K(s, u)=\frac{h_{11} h_{22}-h_{12}^{2}}{g_{11} g_{22}-g_{12}^{2}}
$$

and 
$H(s, u)=\frac{g_{11} h_{11}-2 g_{12} h_{12}-g_{22} h_{22}}{2\left(g_{11} g_{22}-g_{12}^{2}\right)}$,

where $h_{i j}$ are the components of second fundamental form [6].

\section{Main results}

Now we shall give a detailed discussion on null curves on an arbitrary Lorentzian surface in $E_{1}^{3}$. They are defined by the equation

$$
g_{11} d s^{2}+2 g_{12} d s d u+g_{22} d u^{2}=0 \text {. }
$$

When $D=g_{11} g_{22}-g_{12}^{2} \neq 0$ this equation defines two directions, which arrange themselves to the net of null curves. Since the distance of any two points on such curves is zero, they can to be considered as null geodesics. Then

$g_{11}=\left\langle\mathbf{Y}_{s}, \mathbf{Y}_{s}\right\rangle=\left\langle\mathbf{Y}_{s s}, \mathbf{Y}_{s}\right\rangle=0=g_{22}=\left\langle\mathbf{Y}_{u}, \mathbf{Y}_{u}\right\rangle=\left\langle\mathbf{Y}_{\mathrm{u} u}, \mathbf{Y}_{u}\right\rangle, g_{12} \neq 0$.

and

$K(s, u)=-\frac{h_{11} h_{22}}{g_{12}^{2}}+\frac{h_{12}^{2}}{g_{12}^{2}}, H(s, u)=\frac{h_{12}}{g_{12}} \Rightarrow H^{2}-K=\frac{h_{11} h_{22}}{g_{12}^{2}}$.

Hence, we have

$H^{2}=K \Leftrightarrow h_{11} h_{22}=0 \Rightarrow h_{11}=0$ or $h_{22}=0$.

If $h_{11} \neq 0$, then $h_{22}=0$ yields:

$\mathbf{Y}_{\mathrm{u} u}=\mu \mathbf{Y}_{s}+\eta \mathbf{Y}_{u}$,

for some functions $\mu=\mu(s, u)$ and $\eta=\eta(s, u)$. For $g_{11}=g_{22}=0$, we have $\mathbf{Y}_{\mathrm{u} u}=0$. This

shows that $\mathbf{Y}_{u}$ has a fixed direction and all the $u$-curves on $M$ are null lines. This indicates that $M$ is a null scroll.

Theorem 3.1 In Minkowski 3-space, locally, all the Lorentzian surfaces parameterized as surfaces with two families of null geodesics are null scrolls if and only if $H^{2}=K$.

Let

$M: \mathbf{Y}(s, u)=\alpha(s)+u \mathbf{X}(s), u \in \mathbb{R}$,

be a ruled surface with lightlike ruling $\operatorname{inE}_{1}^{3}$. This means that $\boldsymbol{\alpha}(s), \mathbf{X}(s) \in E_{1}^{3}$ and $\left\langle\mathbf{X}, \mathbf{X}>=<\mathbf{X}, \mathbf{X}^{\prime}>=0, s \in I, I\right.$ is open interval. The tangent space to $M$ at an arbitrary point $\mathbf{Y}(s, u)$ is spanned by

$$
\mathbf{Y}_{s}=\boldsymbol{\alpha}^{\prime}(s)+u \mathbf{X}^{\prime}(s), \mathbf{Y}_{u}=\mathbf{X} \text {. }
$$

Therefore, the matrix $\left(g_{i j}\right)$ of the induced metric on $M$ reads as follows:

$$
\left(g_{i j}\right)=\left(\begin{array}{cc}
\left\|\boldsymbol{\alpha}^{\prime}\right\|^{2}+2 u & <\boldsymbol{\alpha}^{\prime}, \mathbf{X}^{\prime}>+u^{2}\left\|\mathbf{X}^{\prime}\right\|^{2}<\boldsymbol{\alpha}^{\prime}, \mathbf{X}> \\
<\boldsymbol{\alpha}^{\prime}, \mathbf{X}> & 0
\end{array}\right) .
$$

From this we see that $D=g_{11} g_{22}-g_{12}^{2}=-g_{12}^{2}=-\left\langle\boldsymbol{\alpha}^{\prime}(s), \mathbf{X}\right\rangle^{2}$. When $\left\langle\boldsymbol{\alpha}^{\prime}(s), \mathbf{X}>\neq 0\right.$ the surface $M$ is non-degenerate and Lorentzian. When $\left\langle\boldsymbol{\alpha}^{\prime}(s), \mathbf{X}\right\rangle=0$ the surface $M$ is degenerate. In this case, we can indeed determine $u$ as a function of $s$ in such away that the curve $\mathbf{Y}=\mathbf{Y}(s, u(s))$ is tangent to $\mathbf{X}$. Then, the tangent vector of this curve must in this case have the direction of the $\mathbf{X}$ :

$\mu(s) \mathbf{X}=\alpha^{\prime}+u^{\prime} \mathbf{X}+u \mathbf{X}^{\prime}$,

for some function $\mu=\mu(s)$. Then vectors $\mathbf{X}^{\prime}, \mathbf{X}$ and $\boldsymbol{\alpha}^{\prime}$ are coplanar, since

$\langle\mathrm{X}, \mathrm{X}\rangle=\left\langle\mathrm{X}, \mathrm{X}^{\prime}\right\rangle=\left\langle\alpha^{\prime}, \mathrm{X}\right\rangle=0$, we find

$u(s)=-\frac{\left\langle\alpha^{\prime}, \alpha^{\prime}\right\rangle}{\left\langle\alpha^{\prime}, \mathbf{X}^{\prime}\right\rangle}=-\frac{\left\langle\alpha^{\prime}, \mathbf{X}^{\prime}\right\rangle}{\left\|\mathbf{X}^{\prime}\right\|^{2}}$,

except for the case that $\mathbf{X}^{\prime}=0$, when we have a Lorentzian cylinder. Substitution of this value of $u$ in Eq. (3.1) gives us the edge of regression of $\mathrm{M}$. This curve, having null tangent, is a null curve. When it shrinks to a point, the surface is a Lorentzian cone. 
Theorem 3.2 In Minkowski 3-space, locally, all the ruled surfaces with lightlike rulings for which $g_{11} g_{22}-g_{12}^{2}=0$ are null developables, that is, they are Lorentzian planes, Lorentzian cylinders, Lorentzian cones, or Lorentzian tangential surfaces.

Assume now that we have chosen $M$ be the ruled surface whose directrix $\alpha(s)$ and rulings $\mathbf{X}(s)$ both are null and $\left\langle\alpha^{\prime}(s), \mathbf{X}>\neq 0\right.$ ( $M$ is non-degenerate) Therefore, from the Frenet equations (2.3) we have:

$$
\mathbf{Y}_{s}=\frac{\partial y}{\partial s}=\mathbf{T}+u\left(-k_{1} \mathbf{X}+k_{3} \mathbf{Z}\right), \mathbf{Y}_{u}=\frac{\partial y}{\partial u}=\mathbf{X}
$$

Hence, the elements of the first fundamental form are given by

$$
g_{11}=2 u k_{1}+u^{2} k_{3}^{2}, g_{12}=-1, g_{22}=0
$$

Therefore, the induced metric is Lorentzian metric and $M$ is a Lorentzian surface. The Gauss map can be directly obtained from $\mathbf{Y}_{s} \times \mathbf{Y}_{u}$ getting

$$
\mathbf{G}(s, u)=\mathbf{Z}(s)+u k_{3}(s) \mathbf{X}(s) \text {. }
$$

It is not difficult to see that shape operator, in the usual frame $\left\{\mathbf{Y}_{s}, \mathbf{Y}_{u}\right\}$, wirtes down as:

$$
S=\left(\begin{array}{cc}
k_{3} & 0 \\
k_{2}+u k_{3}^{\prime} & k_{3}
\end{array}\right)
$$

Thus, the null -scroll ruled surface $M$ has non-diagonalizable shape operator with minimal polynomial $P_{s}(t)=\left(t-k_{3}\right)^{2}$ where $k_{3}$ is the principal curvature along the ruling.It has mean and Gaussian curvatures $H=k_{3}$ and $K=k_{3}^{2}$, respectively. The mean curvature $H$ and the Gaussian curvature $K$ are functions of the arc length parameter and satisfies that $H^{2}=K$ everywhere. For the B-scroll, the Gauss curvature $K$ and the mean curvature $H$ are constant. It turned out that, apart from the umbilical cases, there also exist null scrolls with $H^{2}=K=$ const., which are called generalized umbilical surfaces by Magid [6], and null scrolls with $H^{2}=K \neq$ const. Obviously, these two types of null scrolls have no counterparts in Euclidean space.

Now, it seems natural to pose the following question: Are there other Lorentzian sur-faces, a part from null scrolls satisfying $H^{2}=K$ in 3-dimensional Minkowski space $E_{1}^{3}$ ?.

The answer is affirmative and can be stated as follows:

Theorem 3.3 Let $M$ be a null scroll whose directrix $\boldsymbol{\alpha}(s)$ and rulings $\mathbf{X}(s)$ both are null with the parametrization (3.1) in Minkowski 3-space $E_{1}^{3}$. The surface $M$ can not be a B-scroll if and only if the conditions (1.1) and (1.2) are satisfied.

Proof. We only have to prove that $k_{3}$ is constant and $k_{2}=0$. Firstly, the matrix $\left(g^{i j}\right)$ reads as follows:

$$
\left(g^{i j}\right)=\left(\begin{array}{cc}
0 & -1 \\
-1 & k_{3}^{2} u^{2}+2 k_{1} u
\end{array}\right) \text {. }
$$

Thus, using (2.5) we show that the Laplacian $\Delta$ of $M$ can be expressed as:

$$
\Delta=2 \frac{\partial^{2}}{\partial s \partial u}+2\left(k_{1}+u k_{3}^{2}\right) \frac{\partial}{\partial u}+\left(2 u k_{1}+u^{2} k_{3}^{2}\right) \frac{\partial^{2}}{\partial u^{2}} .
$$

Then a simple calculation shows that:

$$
\Delta \mathbf{Y}=2 u k_{3}^{2} \mathbf{X}+2 k_{3} \mathbf{Z} \text {. }
$$

On the other hand, we have from (1.1) and (3.1)

$$
\Delta \mathbf{Y}=A \mathbf{Y} \Rightarrow \Delta \mathbf{Y}=A \boldsymbol{\alpha}+u A \mathbf{X} \text {. }
$$

Thus, combining (3.11) and (3.12), we obtain

$$
A \mathbf{X}=2 k_{3}^{2} \mathbf{X} \text {. }
$$

$$
A \alpha=2 k_{3} \mathrm{Z} \text {. }
$$

Differentiating (3.14) with respect to $s$ and using (2.3), we find

$$
A \mathbf{T}=2 k_{3}^{2} \mathbf{T}+2 k_{2} k_{3} \mathbf{X}+2 k_{3}^{\prime} \mathbf{Z} \text {. }
$$

Secondly, we suppose that the Gauss map $M$ satisfies the condition (1.2). Then we have

$$
\Delta \mathbf{G}=2 \frac{\partial^{2} \mathbf{G}}{\partial s \partial u}+2\left(k_{1}+u k_{3}^{2}\right) \frac{\partial \mathbf{G}}{\partial u}+\left(2 u k_{1}+u^{2} k_{3}^{2}\right) \frac{\partial^{2} \mathbf{G}}{\partial u^{2}} .
$$

It follows from (2.3) and (3.7) that 
$\Delta \mathbf{G}=2 k_{3}^{2} \mathbf{Z}+\left(2 k_{3}^{\prime}+u k_{3}^{3}\right) \mathbf{X}$.

On the other hand, from (1.2) and (3.7) we have

$\Delta \mathbf{G}=A\left(\mathbf{Z}+u k_{3} \mathbf{X}\right)$.

Thus, combining (3.17) and (3.18) with (3.13), we obtain

$A \mathbf{Z}=2 k_{3}^{2} \mathbf{Z}+2 k_{3}^{\prime} \mathbf{X}$.

Differentiating (3.19) with respect to $S$ and using (2.3), we find

$k_{3} A \mathbf{T}+k_{2} A \mathbf{X}=2 k_{3}^{3} \mathbf{T}+2\left(k_{2} k_{3}^{2}-k_{1} k_{3}^{\prime}+k_{3}^{\prime \prime}\right) \mathbf{X}+6 k_{3} k_{3}^{\prime} \mathbf{Z}$

from which together with (3.13), and (3.15), we have

$\left(2 k_{2} k_{3}^{2}-k_{1} k_{3}^{\prime}+k_{3}^{\prime \prime}\right) \mathbf{X}-4 k_{3} k_{3}^{\prime} \mathrm{Z}=0$.

Since $\mathbf{X}$ and $\mathbf{Z}$ are linearly independent, we can easily find $k_{3}$ is constant and $k_{2}=0$, and the proof is complete.

By the definition of $M$, the base curve intersects the rulings transversely, then we have to write the equation

$$
\boldsymbol{\alpha}(s)=f(s) \mathbf{X}+k_{3}^{-2} \mathbf{X}^{\prime},
$$

where $f(s)$ is a differentiable function on $M$. If $\left(Y_{1}, Y_{2}, Y_{3}\right)$ are the coordinates of $\mathbf{Y}$, then equations (3.1) and (3.21), yield

$$
-Y_{1}^{2}+Y_{2}^{2}+Y_{3}^{2}=k_{3}^{-2}
$$

From Eqs. (3.8) and (3.22) it can easily seen that:

1) When $k_{2}+u k_{3}^{\prime}=0$, i.e., $k_{2}=0$ and $k_{3}=$ const., $M$ is a (non-B-scroll) piece of Lorentzian sphere with radius $\left|k_{3}\right|^{-1}$ (resp. Lorentzian plane) when $k_{3} \neq 0$ (resp. when $k_{3}=0$ ),

2) When $k_{2}+u k_{3}^{\prime} \neq 0$ and $k_{3}=$ const., i.e., $k_{2} \neq 0$ and $k_{3}=$ const., $M$ is a B-scroll,

3) When $k_{2}+u k_{3}^{\prime} \neq 0$ and $k_{3} \neq$ const., i.e., $k_{3}=k_{3}(s), M$ is a null scroll.

Thus, as a result the following Theorem can be given:

Theorem 3.4 In Minkowski 3-space, locally, all the null scrolls surfaces satisfying $H^{2}=K$ can be classified into the following three cases:

i) A Lorentzian plane or sphere.

ii) A B-scroll,

iii) A null scroll with minimal polynomial of shape operator $P_{s}(t)=\left(t-k_{3}\right)^{2}, k_{3}=$ const.

The results in the study is confirmed by following examples:

Example 1. Let $\boldsymbol{\alpha}(s)=(s, s, 1)$ be a null curve and a null vector field

$\mathbf{X}(s)=(s+1, s-1,2 \sqrt{s})$.

Then, a parametrization of a ruled surface $M$ is given by

$\mathbf{Y}(s, u)=(s, s, 1)+\frac{u}{2}(s+1, s-1,2 \sqrt{s}), \quad s>0$,

It is easy to show that

$k_{1}=k_{2}=0, \quad k_{3}=-\frac{1}{2 \sqrt{s}}, \quad H^{2}=K=\frac{1}{4 s}$.

Therefore, the surface is a null-scroll (see Fig. 1).

Example 2. Let $\boldsymbol{\alpha}(s)=\left(s+1, \frac{\sqrt{2}}{2} s, \frac{\sqrt{2}}{2} s+\sqrt{2}\right)$ be a null curve and a null vector field

$\mathbf{X}(s)=\left(\frac{1}{2} s^{2}+s+1, \frac{\sqrt{2}}{4} s^{2}-\frac{\sqrt{2}}{2}, \frac{\sqrt{2}}{4} s^{2}+\sqrt{2} s+\frac{\sqrt{2}}{2}\right)$.

Then, a parametrization of a ruled surface $M$ is given by

$M: \mathbf{Y}(s, u)=\left(-\frac{\sqrt{2}}{12} s^{3}-\frac{\sqrt{2}}{2}(s+u),-\frac{s^{2}}{2},-\frac{\sqrt{2}}{12} s^{3}+\frac{\sqrt{2}}{2}(s+u)\right)$.

The curvature functions are:

$k_{1}=k_{2}=0, \quad k_{3}=1$. 
Then $M$ is a non-B-scroll surface (see Fig. 2).

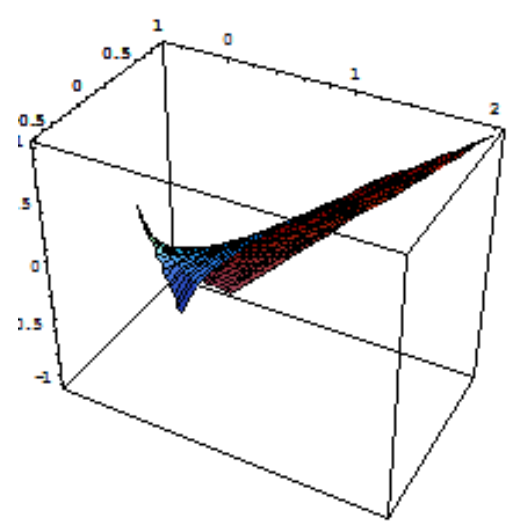

Fig. 1. Null-scroll surface.

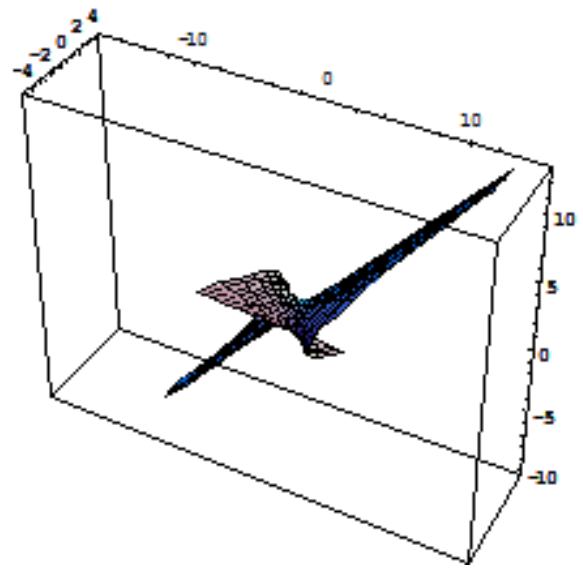

Fig. 2. Non B-scroll surface.

\section{Bibliography}

[1] T. Takahashi. Minimal immersions of Riemannian manifolds, J. Math. Soc. Jpn. 18(1966),380-385.

[2] O. J. Garay. An extension of Takahashi's theorem, Geom. Dedicate 34 (1990) 105-112.

[3] C. Baikoussis and D. E. Blair. On the Gauss map of ruled surfaces, Glasgow Math. J. 34 (1992), 355-359.

[4] S. M. Choi, On the Gauss map of ruled surfaces in a 3-dimensional Minkowski space,Tsukuba J. Math. 19 (1995), $285-304$.

[5] L. J. Alias, A. Ferrandez,P. Lucas, and M. A. Merono. On the Gauss map of B-scrolls. Tsukuba J. Math. 22 (1998), $371-377$.

[6] B. O’Neill. Semi-Riemannian geometry, Academic Press. Inc., 1983.

[7] L. Graves. Codimension one isometric immersions between Lorentz spaces, Trans. Amer. Math. Soc. 252 (1979), 367-392.

[8] K. L. Duggal, A. Bejancu. LightlikeSubmanifolds of Semi-Riemannian Manifolds and Applications, Kluwer Academic Publishers, 1996.

[9] S. M. Choi,U. H. Ki and Y. J. Suh. On the Gauss map of null scrolls, Tsukuba J. Math. 22 (1998), 273-279.

[10] L. K. Graves. Codimension one isometric immersions between Lorentz spaces, Trans. Amer. Math. Soc. 252 (1979), $367-392$.

[11] M. Magid. Lorentzian isoparametric hypersurfaces, Pacific J. Math. 118 (1) (1985), 165-197.

[12] Ji Fenghui, Zhong H. H. On Lorentzian surfaces with $\mathrm{H}^{2}=\mathrm{K}$ in Minkowski 3-space, J. Math. Anal. Appl. 334 (2007), $54-58$. 